\title{
Análise econômica da redução de incertezas geológicas de reservatórios de petróleo
}

\author{
Eliana Luci Ligero ${ }^{1}$, Valmir Francisco Risso ${ }^{I}$ \& Denis José Schiozer ${ }^{I}$
}

\begin{abstract}
Resumo Reservatórios de petróleo, nas fases de avaliação e desenvolvimento, possuem elevado número de incertezas geológicas, que influenciam significativamente na recuperação de óleo e gás. Tais incertezas podem ser reduzidas ou até mesmo eliminadas pela obtenção de informações, que são fundamentais para mitigar o risco envolvido em projetos, mas em contrapartida possuem um custo associado. A viabilidade econômica da obtenção de uma ou mais informações adicionais (completa ou incompleta) pode ser quantificada pelo Valor de Informação (VDI). A metodologia de cálculo do VDI apresentada neste trabalho baseia-se na combinação dos atributos geológicos incertos através da árvore de derivação, onde cada ramo da árvore representa um modelo de simulação do reservatório. A quantificação do VDI, no caso ideal, deveria considerar os benefícios que poderiam ser obtidos pelo emprego das estratégias de produção mais adequadas para cada um dos possíveis cenários geológicos. Na prática, a determinação de uma estratégia de produção otimizada para cada modelo da árvore é inviável, pois o número de modelos cresce exponencialmente com o aumento do número de atributos incertos. Deste modo, as incertezas geológicas são representadas pelos Modelos Geológicos Representativos (MGR) - modelos selecionados por um procedimento definido e usados como base para a determinação de diferentes estratégias de produção, a fim de considerar os benefícios de informações adicionais. No exemplo usado neste trabalho, um poço adicional foi perfurado em um reservatório de óleo na fase de desenvolvimento, com elevado grau de incertezas geológicas, para redução da incerteza e estudo da viabilidade econômica da obtenção da informação que foi avaliada pelo cálculo do VDI.
\end{abstract}

Palavras-chave: Incertezas geológicas, modelos geológicos representativos, valor de informação.

\begin{abstract}
Economic feasibility of reduction of geological uncertainties in petroleum reservoir. Petroleum reservoirs in appraisal and development phases have high number of geological uncertainties with strong influence in oil and gas recovery. These uncertainties can be reduced or eliminated through additional information acquisitions that are fundamental to mitigate the risk of projects; however, the acquisition of new information has an associated cost. The economic viability to obtain one or more information (complete or incomplete) can be quantified by the Value of Information (VoI). The methodology to VoI calculation used in this work is based on the combination of uncertain geological attributes by the derivative tree technique, where each branch of the tree represents a reservoir simulation model. The VoI quantification, in the ideal case, should consider the benefits that could be obtained using the most adequate production strategy for each possible geological scenario. In practice, it is not viable to optimize the production strategy of each model that constitutes the derivative tree. Therefore, the geological uncertainties are represented by Geological Representative Models (MGR), which are used as base to determine different production strategies in order to consider the benefits of additional information. The studied case is represented by an oil reservoir in development phase with high level of uncertainties and one additional well is drilled in reservoir in order to reduce the uncertainties and to study the economic feasibility to obtain additional information, which was evaluated by VoI calculation.
\end{abstract}

Keywords: Geological uncertainties, geological representative models, value of information.

INTRODUÇÃO Incertezas estão presentes em campos de petróleo nas fases de avaliação e desenvolvimento e são resultantes, principalmente, do pouco conhecimento associado à caracterização geológica dos reservatórios, tais como, volume in situ, continuidade, falhas e propriedades de rochas e fluidos. Outras fontes comuns de incertezas são representadas por fatores tecnológicos associados a dúvidas sobre o avanço da tecnologia com o tempo; aspectos econômicos, principalmente o preço do óleo e os custos e; por fatores polí- ticos, como por exemplo, o regime fiscal adotado.

Este cenário de incertezas exerce um forte impacto nos resultados econômicos dos projetos e isso é mais crítico em campos marítimos, onde os investimentos são elevados. Begg et al.(2002) afirmam que o valor de um projeto é fortemente influenciado pelas incertezas, e o baixo investimento destinado ao processo de tomada de decisão resulta num baixo desempenho da indústria de exploração e produção de petróleo (E\&P). Deste modo, a indústria de E\&P está sempre associada 
a um risco, seja este em maior ou menor intensidade. O risco, segundo Stermole \& Stermole (1974), pode ser definido como a avaliação de um investimento usando um mecanismo que incorpore as probabilidades de ocorrência de sucesso ou falha e diversos valores de parâmetros de investimento. Três tipos de riscos podem ser considerados no processo de tomada de decisão relacionado a um campo de petróleo na fase de desenvolvimento: (1) a perda de oportunidade quando o prospecto é tido como não econômico e abandonado, mas na realidade é economicamente viável, (2) o desenvolvimento não comercial, ou seja, um campo é não econômico, porém considerado como econômico e (3) desenvolvimento sub-ótimo quando o campo produz menos que o máximo retorno econômico esperado, o qual seria obtido se o modelo correto de reservatório tivesse sido considerado (Demirmen, 2001). Uma maneira possível de reduzir o risco envolvido na fase de desenvolvimento é através da obtenção de informações adicionais, que podem reduzir determinada incerteza ou até mesmo eliminar por completo a incerteza de um determinado atributo. Embora informações adicionais sejam extremamente úteis na redução de incertezas, as mesmas estão associadas a um custo, que em alguns casos pode ser inviável economicamente. Deste modo, Mian (2002) sugere que antes da aquisição de qualquer informação adicional duas questões sejam consideradas: se a obtenção da informação adicional vale a pena e se várias fontes potenciais de informação são capazes de melhorar o processo de decisão, qual delas é a preferida.

VALOR DE INFORMAÇÃO A viabilidade econômica da obtenção de uma ou mais informações adicionais pode ser quantificada pelo Valor de Informação (VDI). O cálculo do VDI pode ser realizado pela diferença entre o Valor Monetário Esperado (VME) com e sem informação (Demirmen, 1996 e 2001). A análise e cálculo do VDI são usualmente descritos por árvores de derivação e decisão, mas podem ser explorados por outros métodos, tais como, Monte Carlo e Teoria das Opções Reais.

A análise do VDI pode ser interpretada como um critério consistente para tomada de decisão, reduzindo o risco econômico na fase de desenvolvimento. A relevância e aplicabilidade da análise do VDI no processo de decisão e análise de risco de projetos de E\&P foi apresentada por vários autores (Clemen, 1995; Koninx, 2000; Floris \& Peersmann, 2000; Coopersmith \& Cunninghan, 2002). Hayashi et al. (2007) demonstraram que além do valor da informação, a redução do risco deve ser considerada no processo de decisão associado à aquisição de uma informação.

$\mathrm{Na}$ literatura técnica disponível, não há metodologias claras e bem definidas para o cálculo de VDI em casos complexos com muitos atributos incertos nas fases de avaliação e desenvolvimento. De modo análogo, procedimentos sistemáticos que explorem o potencial da metodologia de cálculo do VDI, principalmente se a simulação numérica de reservatórios é empregada como ferramenta de cálculo são inexistentes. Na indústria petrolífera, problemas complexos são demasiadamente simplificados, desde que é prática comum considerar apenas três modelos representativos (otimista, provável e pessimista), resultando num valor de informação subestimado. De fato, Xavier (2004) mostrou que na avaliação do VDI, o Método DSS utilizado por Floris \& Peersmann (2000) e o emprego de apenas três modelos representativos sub-avaliam o VDI. Portanto, na quantificação do VDI deve-se considerar a possibilidade de cada um dos vários cenários possíveis de um determinado reservatório possuir uma estratégia de produção específica após a obtenção da informação.

Uma informação pode ser classificada como completa ou incompleta, perfeita ou imperfeita. Uma informação é considerada completa e perfeita quando a incerteza é eliminada, de modo a não exercer mais influência sobre o risco e o Valor Monetário Esperado (VME) do projeto. Uma informação incompleta não elimina toda a dúvida relacionada a um atributo, apenas reduz o nível de incerteza. Além de incompleta, uma informação pode ser imperfeita, se não houver confiabilidade total no valor medido. Uma explicação detalhada sobre informações completas e incompletas foi apresentada por Xavier et al. (2006).

A metodologia de cálculo do VDI proposta por Xavier (2004) foi empregada em reservatórios simples e reais, sendo admitidas tanto informações completas, quanto informações incompletas. A consideração de informação completa e perfeita a respeito da incerteza no modelo estrutural de um reservatório simples foi utilizada para exemplificar o procedimento de cálculo de VDI. No cálculo do VDI do campo real de Namorado foram consideradas as seguintes situações: dois atributos incertos passíveis de redução de incerteza e informação completa e perfeita para os dois atributos, quatro atributos incertos passíveis de redução de incerteza e informação completa e perfeita para os todos os atributos e quatro atributos incertos passíveis de redução de incerteza e informação completa e perfeita para três atributos e informação incompleta para o quarto atributo (Xavier, 2004). Esta mesma metodologia foi empregada com sucesso por Hayashi et al. (2007) no cálculo do VDI de um modelo sintético baseado num campo marítimo típico da Bacia de Campos.

METODOLOGIA A quantificação do VDI durante as fases de avaliação e desenvolvimento de campos de petróleo é realizada por uma metodologia nova e confiável, que se aplica tanto a modelos simples como complexos, caracterizados por poucos ou muitos atributos geológicos incertos (Xavier, 2004). As etapas iniciais do cálculo do VDI correspondem às etapas da metodologia de análise de risco denominada de árvore de derivação e proposta por Steagall \& Schiozer (2001) e Schiozer et al. (2004). Os passos a serem seguidos no cálculo do VDI são descritos a seguir.

Etapa 1: Definição das Incertezas Seleção de todos os atributos incertos, definição de seus níveis de incer- 
teza e probabilidades de ocorrência associada a cada nível. O usual é considerar inicialmente três níveis de incerteza para cada atributo: provável, otimista e pessimista. A discretização em três níveis pode ser uma boa representação inicial para os atributos contínuos, sendo que quanto maior a importância do atributo, maior o número de níveis em que o atributo deve ser discretizado. Os atributos caracterizados por distribuições discretas de probabilidades têm seus níveis de incerteza definidos pela qualidade das análises confiáveis e representativas disponíveis.

Etapa 2: Modelo Base Elaboração do modelo geológico constituído por todos os atributos incertos com seus valores mais prováveis. Tal modelo, denominado de modelo base, tem a conotação de modelo determinístico. A estratégia de produção deste modelo é selecionada em termos de uma função-objetivo pré-estabelecida, como por exemplo, o Valor Presente Líquido (VPL). Esta etapa tem como objetivo, portanto, a determinação de um modelo do reservatório com uma estratégia de produção adequada para elaboração da curva de risco.

Etapa 3: Análise de Sensibilidade A análise de sensibilidade visa à seleção dos atributos incertos mais críticos de modo a reduzir o número de atributos a serem considerados no processo de quantificação do risco e conseqüentemente no cálculo do VDI. O procedimento adotado na análise de sensibilidade consiste na variação de um atributo incerto por vez no modelo base. O VPL representa a principal função-objetivo, na qual o efeito dos atributos incertos é analisado, contudo funções tais como, produção acumulada de óleo $(\mathrm{Np})$ e de água (Wp) devem ser levadas em consideração, pois atributos com pouco efeito no VPL podem causar variações significativas na produção de óleo ou água.

Etapa 4: Árvore de Derivação Elaboração da árvore de derivação, na qual cada ramo corresponde a um modelo de simulação completo e distinto. O número de atributos a serem incluídos na área de derivação depende dos objetivos do caso em estudo. O procedimento da adição gradativa de atributos críticos na árvore de derivação (Costa \& Schiozer, 2003) garante que apenas os atributos mais importantes constituam a árvore e que uma precisão aceitável seja obtida sem excessivo tempo computacional.

Etapa 5: Curva de Risco Os vários cenários gerados pela combinação dos atributos críticos através da árvore de derivação são submetidos à simulação numérica de fluxo. Os resultados das simulações são empregados no cálculo do VPL de cada um dos modelos. Os VPL obtidos são tratados estatisticamente a fim de elaborar a curva de risco e de quantificar o risco envolvido no projeto. É importante observar que a elaboração da curva de risco não é necessária no cálculo do VDI, porém de acordo com Hayashi (2006), sua importância é fundamental na determinação qualitativa ou quantitativa do risco envolvido no projeto, o que pode também influen- ciar a decisão de adquirir ou não uma nova informação (isso não foi feito neste trabalho, pois o VDI foi baseado somente no cálculo descrito até a Etapa 12).

Etapa 6: Aquisição de Informação Verifica-se a possibilidade de redução ou eliminação das incertezas de determinados atributos incertos através da aquisição de novas informações. O investimento em informações adicionais sobre o reservatório pode estar associado a operações, tais como, realização de sísmica 4D, perfuração de poços de delimitação, execução de testes de pressão e/ou de laboratório e implantação de um plano piloto de produção.

Etapa 7: Árvore de Decisão A árvore de derivação é transformada em árvore de decisão após a aquisição da informação adicional. A figura 1 ilustra a transformação da árvore de derivação em árvore de decisão. Observase que o nó de um evento com probabilidade de ocorrer com incerteza (representado por um círculo) é transformado em um nó de decisão (representado por um quadrado). $\mathrm{O}$ triângulo horizontal representa que não há decisões a serem tomadas ou ocorrência de eventos. Na metodologia para o cálculo do VDI, a definição de grupo é importante. De acordo com a figura 1, a árvore de derivação é constituída por um único grupo e uma única estratégia de produção e a árvore de decisão, por sua vez, é composta por três grupos sendo que para cada grupo, uma estratégia de produção distinta pode ser considerada.

\section{Etapa 8: Seleção dos Modelos Geológicos Represen-} tativos (MGR) Os modelos geológicos representativos (MGR) visam representar as incertezas geológicas do reservatório e variações de estratégias de produção. A seleção dos MGR, segundo Xavier (2004) depende dos seguintes pontos: (1) do número de atributos e seus respectivos níveis passíveis de redução de incerteza quanto maior o número desses atributos, maior o número de MGR a ser selecionado; (2) da dispersão apresentada no gráfico do Valor Presente Líquido versus Fator de Recuperação de Óleo - quanto maior a dispersão dos pontos, maiores as possíveis diferenças nas estratégias de produção dos MGR; (3) das probabilidades de ocorrência de cada modelo - altas probabilidades de ocorrência concentradas em determinados modelos devem ser diluídas em um número maior de modelos; (4) da precisão desejada no processo - quanto maior o número

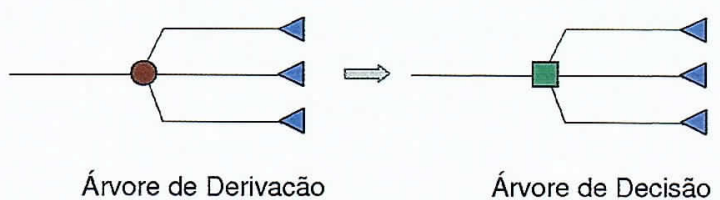

Figura 1 - Exemplo de transformação de árvore de derivação em árvore de decisão (Xavier, 2004). 
de MGR, maior a precisão e confiabilidade no cálculo e; (5) do tempo disponível para a realização do cálculo do valor da informação. É importante ressaltar que o modelo base deve ser considerado como um MGR.

Portanto, os MGR são selecionados visando definir diferentes estratégias de produção para cada cenário ou grupo da árvore de decisão, de modo que quanto maior o número de MGR selecionados, maior é a garantia de otimização da estratégia de produção para o grupo. O uso de um número limitado de MGR é baseado apenas na intenção de diminuir o tempo computacional da análise.

Etapa 9: Otimização das Estratégias de Produção dos MGR Os MGR são submetidos ao processo de otimização de suas estratégias de produção. Um procedimento possível para otimização de estratégias foi proposto por Santos \& Schiozer (2003). É necessário que as estratégias de produção dos MGR e do modelo base estejam otimizadas, caso as mesmas não estejam é necessário otimizá-las. Deste modo, o procedimento de otimização pode ser iterativo.

Etapa 10: Cálculo do Valor Monetário Esperado sem Informação O cálculo do Valor Monetário Esperado sem informação $\left(\mathrm{VME}_{\mathrm{SI}}\right)$ é realizado através da equação 1 . Todos os modelos da árvore de derivação são simulados considerando cada uma das estratégias de produção otimizadas dos MGR a fim de determinar a estratégia que maximiza o VME do caso sem informação. Conseqüentemente, o número de modelos a ser simulado é igual ao número de modelos MGR multiplicado pelos ramos da árvore de derivação (número total de cenários).

$V M E_{S I}=\underset{w=1}{n_{E S T}}\left\{\sum_{j=1}^{N}\left[\left(V P L_{\text {estrategia }(w), \text { modelo }}\right) \cdot P_{\text {modelo },}\right]\right\}$

onde: $\mathrm{n}_{\text {EST }}$ é o número de estratégias otimizadas,

w é índice que varia de 1 a $\mathrm{n}_{\text {EST }}$, N é número total de modelos ou cenários possíveis e jé um índice que varia de 1 a N.

Etapa 11: Cálculo do Valor Monetário Esperado com Informação O Valor Monetário Esperado com Informação $\left(\mathrm{VME}_{\mathrm{CI}}\right)$ é obtido pela equação 2 , generalizada para $w$ estratégias.

$V M E_{C I}=\sum_{k=1}^{G}\left\{\underset{w=1}{m_{E S T} x}\left[\sum_{\ell=1}^{N_{G}}\left(V P L_{\text {estrategia }(w), \text { modelo }}\right) \cdot P_{\ell}\right]\right\}_{(k)}$

onde: $\mathrm{N}_{\mathrm{G}}$ é o número de modelos ou cenários por grupo, $\ell$ é um índice que varia de $1 \mathrm{a} \mathrm{N}_{\mathrm{G}} \mathrm{G}$ é número de grupos da árvore e k é o índice que varia de 1 a G.

É importante ressaltar que, na Etapa 10, uma única estratégia deve ser escolhida, aquela que maximiza o VME de toda a árvore de derivação. Por outro lado, na Etapa 11, cada grupo terá uma estratégia que maximiza o VME do grupo. Além disso, a equação generalizada para o cálculo do VME é dada pela equação 3.

$$
V M E=\sum_{k=1}^{G}\left\{\underset{w=1}{n_{E S T} \dot{x} x}\left[\sum_{\ell=1}^{N_{G}}\left(V P L_{\text {estratégia }(w), \bmod e l o_{\ell}}\right) \cdot P_{\ell}\right]\right\}_{(k)}
$$

Portanto, a equação 3 reduz-se a equação do $\mathrm{VME}_{\mathrm{SI}}$, se $\mathrm{G}=1$ e $\mathrm{N}_{\mathrm{G}}=\mathrm{N}$. Para $\mathrm{G}>1$, a equação 3 reduz-se a equação do $\mathrm{VME}_{\mathrm{Cl}}$.

Etapa 12: Cálculo do Valor de Informação $O$ Valor da Informação é obtido pela diferença entre o $\mathrm{VME}_{\mathrm{CI}} \mathrm{e}$ o $\mathrm{VME}_{\mathrm{SI}}$ :

$V D I=V M E_{C l}-V M E_{S I}$

O fluxograma do procedimento de cálculo do VDI encontra-se na figura 2.

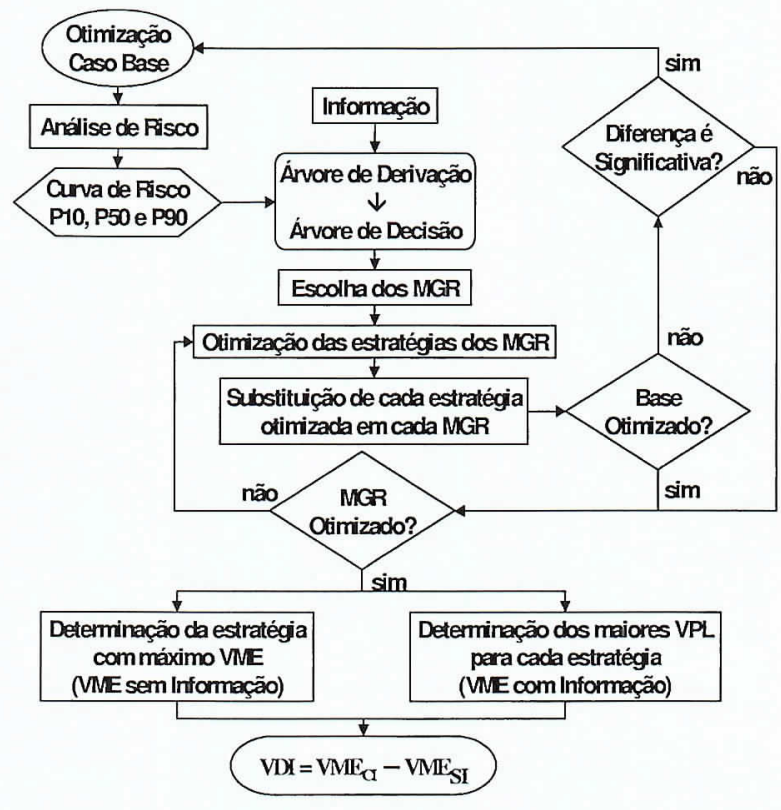

Figura 2 - Representação esquemática do método de cálculo do VDI (Xavier, 2004).

APLICAÇÃo O caso estudado é uma modificação do modelo sintético proposto por Maschio et al. (2008). O reservatório encontra-se na fase avaliação e desenvolvimento e possui algumas incertezas associadas. A rocha reservatório é constituída por dois tipos de fácies, denominados de reservatório e de folhelho, ambos com incertezas associadas. Todos os atributos incertos foram constituídos por três níveis de incerteza: provável (nível 0), pessimista (nível 1) e otimista (nível 2) e foram admitidos como independentes entre si (Tab. 1). Portanto, inicialmente dez atributos do reservatório foram considerados como incertos.

O modelo de simulação do reservatório foi representado por uma malha com dimensões $25 \times 25 \times 34$, 
Tabela I - Atributos incertos e suas probabilidades de ocorrência.

\begin{tabular}{|c|c|c|}
\hline Atributos & Níveis & Probabilidade \\
\hline Contato óleo-água & $\begin{array}{l}d w o c 0 \\
d w o c 1 \\
d w o c 2\end{array}$ & $\begin{array}{l}0,6 \\
0,2 \\
0,2\end{array}$ \\
\hline $\begin{array}{l}\text { Compressibilidade da } \\
\text { Rocha }\end{array}$ & $\begin{array}{l}\text { cpor0 } \\
\text { cporl } \\
\text { cpor } 2\end{array}$ & $\begin{array}{l}0,6 \\
0,2 \\
0,2 \\
\end{array}$ \\
\hline Porosidade - Folhelho & $\begin{array}{l}\text { porF0 } \\
\text { porFl } \\
\text { porF2 }\end{array}$ & $\begin{array}{l}0,6 \\
0,2 \\
0,2\end{array}$ \\
\hline $\begin{array}{l}\text { Permeabilidade } \\
\text { Horizontal - Folhelho }\end{array}$ & $\begin{array}{l}k x F 0 \\
k x F 1 \\
k x F 2\end{array}$ & $\begin{array}{l}0,6 \\
0,2 \\
0,2\end{array}$ \\
\hline $\begin{array}{l}\text { Permeabilidade } \\
\text { Vertical - Folhelho }\end{array}$ & $\begin{array}{l}k z F 0 \\
k z F 1 \\
k z F 2\end{array}$ & $\begin{array}{l}0,6 \\
0,2 \\
0,2\end{array}$ \\
\hline $\begin{array}{l}\text { Permeabilidade } \\
\text { Relativa Água/Óleo - } \\
\text { Folhelho }\end{array}$ & $\begin{array}{l}k r F 0 \\
k r F 1 \\
k r F 2\end{array}$ & $\begin{array}{l}0,6 \\
0,2 \\
0,2\end{array}$ \\
\hline $\begin{array}{l}\text { Porosidade - } \\
\text { Reservatório }\end{array}$ & $\begin{array}{l}\text { porR0 } \\
\text { porR1 } \\
\text { porR2 }\end{array}$ & $\begin{array}{l}0,6 \\
0,2 \\
0,2\end{array}$ \\
\hline $\begin{array}{l}\text { Permeabilidade } \\
\text { Horizontal - } \\
\text { Reservatório }\end{array}$ & $\begin{array}{l}k x R 0 \\
k x R 1 \\
k x R 2\end{array}$ & $\begin{array}{l}0,6 \\
0,2 \\
0,2\end{array}$ \\
\hline $\begin{array}{l}\text { Permeabilidade } \\
\text { Vertical - Reservatório }\end{array}$ & $\begin{array}{l}k z R 0 \\
k z R 1 \\
k z R 2 \\
\end{array}$ & $\begin{array}{l}0,6 \\
0,2 \\
0,2 \\
\end{array}$ \\
\hline $\begin{array}{l}\text { Permeabilidade } \\
\text { Relativa Água/Óleo - } \\
\text { Reservatório }\end{array}$ & $\begin{array}{l}k r R 0 \\
k r R 1 \\
k r R 2\end{array}$ & $\begin{array}{l}0,6 \\
0,2 \\
0,2\end{array}$ \\
\hline
\end{tabular}

onde: (0), (1) e (2) são os níveis provável, pessimista e otimista, respectivamente; os termos otimista e pessimista são em relação ao valor do atributo e não ao impacto que eles exercem sobre a função-objetivo.

constituindo um total de 21.250 blocos. As simulações foram executadas num simulador comercial do tipo Black-Oil, num período de tempo de 20 anos (7300 dias). Os mapas de porosidade e permeabilidade absoluta do modelo base (constituído pelos atributos mais prováveis) são apresentados nas figuras 3 e 4 , respectivamente. As regiões de porosidade 5\% correspondem a fácies folhelho, que possuem permeabilidade absoluta da mesma ordem de grandeza da fácies reservatório.

Um atributo passível de redução de incerteza, através da perfuração de um poço adicional, é o contato água-óleo $(d w o c)$. Foi assumido que a informação sobre tal atributo é completa e perfeita, portanto, elimina completamente a dúvida quanto ao contato água-óleo. A figura 5 ilustra os três níveis de incerteza do atributo contato água-óleo.

Duas situações distintas, quanto o modo de operação dos poços produtores do modelo base, foram consideradas para o modelo geológico representado nas figuras 3, 4 e $5 \mathrm{com}$ as incertezas descritas na tabela

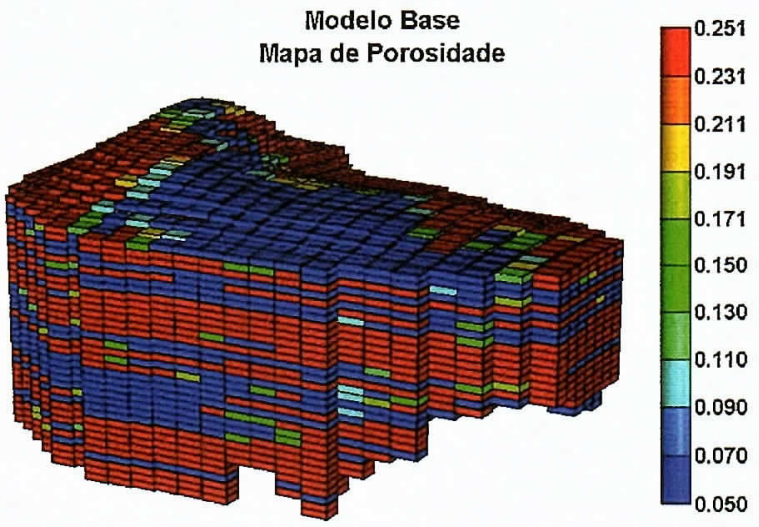

Figura 3 - Mapa de porosidade do modelo base.

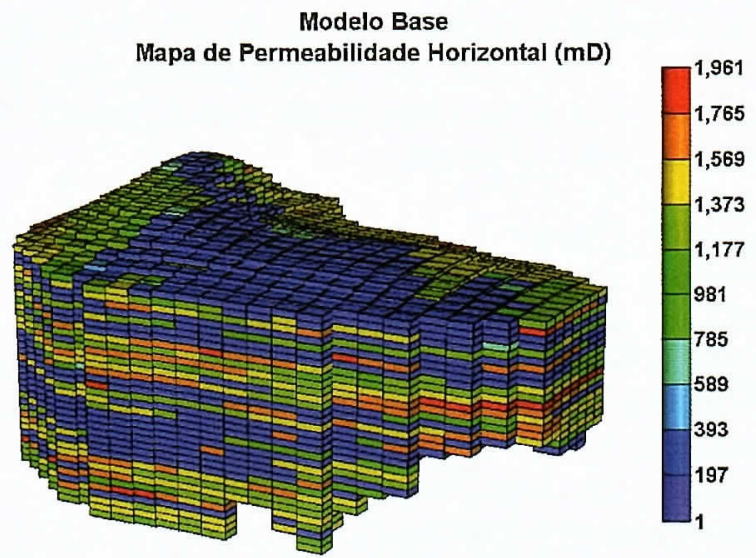

Figura 4 - Mapa de permeabilidade absoluta horizontal do modelo base.

1. Numa primeira situação, denominada de Caso 1, os poços produtores foram monitorados por camada, de modo que quando a fração de água produzida atingisse o valor de $90 \%$ numa determinada camada, a produção na mesma seria fechada. No Caso 2 , a condição de operação dos poços produtores foi mais flexível, sendo que não foi realizado o monitoramento da fração de água nas camadas em que os produtores estavam completados. Esta diferença nas condições de operação dos produtores, embora possa ser considera pequena, resultou em diferentes modelos bases com estratégias de produção distintas para os Casos 1 e 2.

RESULTADOS Independente da condição de operação dos poços produtores, os Casos 1 e 2 apresentaram incerteza em 10 atributos conforme descrito na tabela 1. O emprego de todos esses atributos incertos na obtenção da curva de risco seria inviável, pois seria necessário simular 59.049 modelos, que equivale $3^{10}$, pois todos os atributos possuem 3 níveis de incerteza.

Análise de Sensibilidade A análise de sensibilidade em ambos os casos foi realizada tomando-se como base as funções-objetivo Valor Presente Líquido (VPL) e 


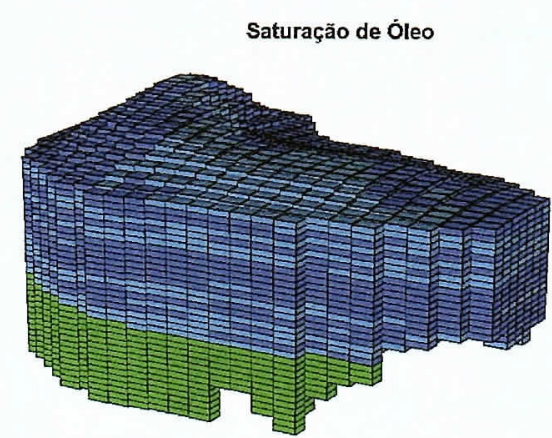

(a)

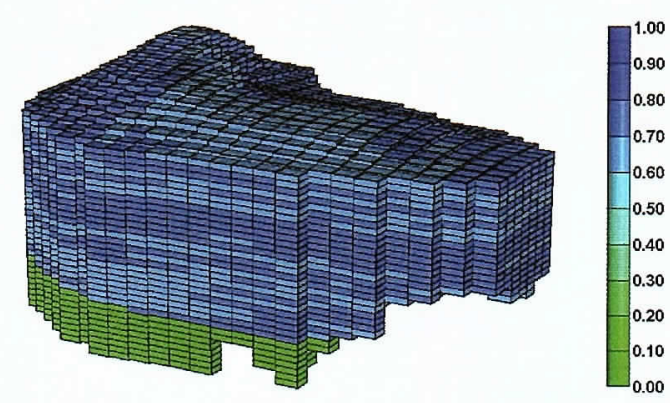

(b)

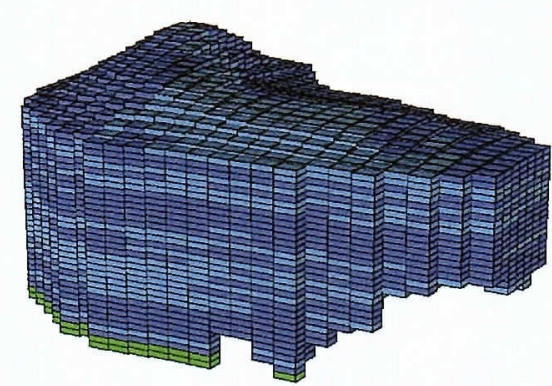

(c)

Figura 5 - Niveis de incerteza do atributo contato água-óleo: (a) pessimista, (b) provável e (c) otimista.

Produção Acumulada de Óleo (Np). A figura 6 ilustra a análise de sensibilidade para o Caso 1. Observa-se que tanto para a função-objetivo VPL, quanto para a função $\mathrm{Np}$, os atributos que se apresentaram mais críticos foram $d w o c$ (contato óleo-água), $k x R$ (permeabilidade absoluta horizontal na fácies reservatório) e porR (porosidade na fácies reservatório). Para esses três atributos, ambos os níveis otimista e pessimista mostraram efeitos significativos nas funções analisadas.

$O$ fato do modelo base do Caso 2 ser distinto do modelo do Caso 1 fez com que a análise de sensibilidade para o Caso 2 se mostrasse diferente da análise de sensibilidade do Caso 1, ainda que os mesmos atributos incertos e as mesmas funções-objetivo VPL e $\mathrm{Np}$ tenham sido consideradas (Fig. 7). Observa-se através das funções VPL e Np, de modo análogo ao Caso 1 , que os atributos mais críticos foram $d w o c$ (contato óleo-água), $k x R$ (permeabilidade absoluta horizontal na fácies reservatório) e por $R$ (porosidade na fácies reservatório). Para esses três atributos, ambos os níveis otimista e pessimista mostraram efeitos significativos nas

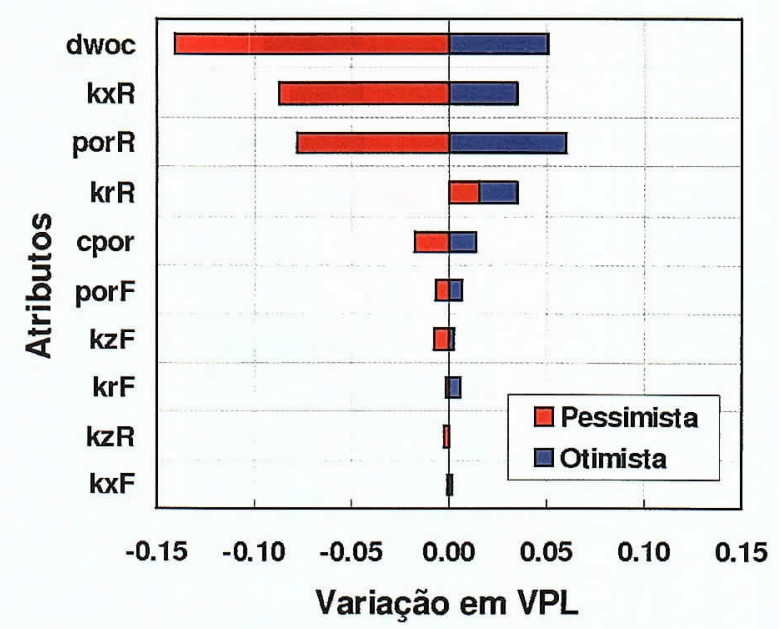

(a)

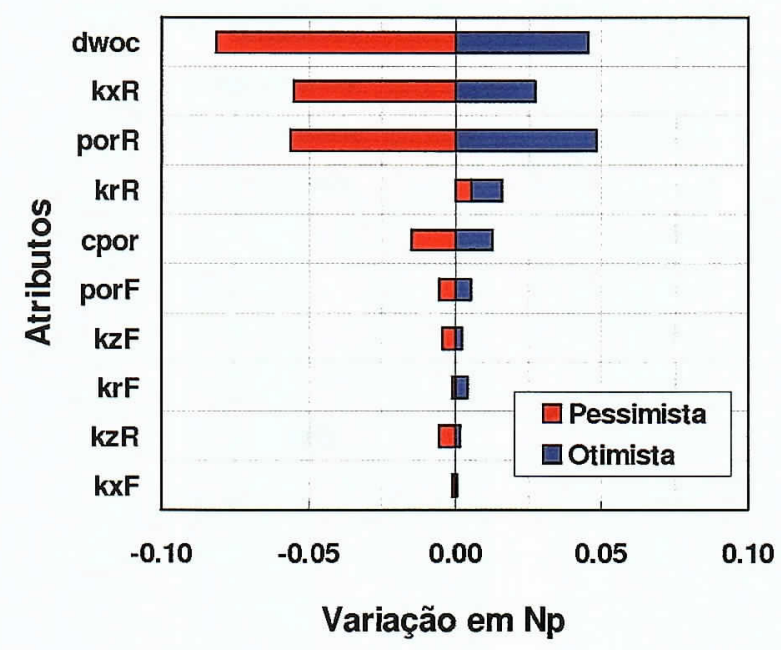

(b)

Figura 6-Caso 1: Análise de sensibilidade para as funções-objetivo. (a) VPL e (b) Np.

funções analisadas. Contudo, o atributo $k z R$ (permeabilidade absoluta vertical na fácies reservatório) apresentou efeito significativo para a função VPL apenas para seu nível otimista.

Atributos Críticos e Curvas de Risco O número total de atributos críticos a ser considerado na elaboração da curva de risco, em ambos os casos, foi determinado de acordo com o critério da adição gradativa de atributos à árvore de derivação.

A figura 8 ilustra o efeito da adição gradativa na curva de risco do Caso 1. É importante ressaltar que todos os níveis (otimista, provável e pessimista) foram utilizados para todos os atributos: $d w o c, p o r R, k x R, k r R$ e cpor. A adição do atributo cpor resultou numa variação pouco significativa na curva de risco comparada à curva com 4 atributos ( $d w o c, p o r R, k x R$ e $k r R$ ). Este fato pôde ser observado, também, através da figura 9, a qual mostra que a adição do atributo cpor praticamente estabilizou os valores dos percentis P10 (otimista), P50 (provável) e P90 (pessimista). Deste modo, a adição 


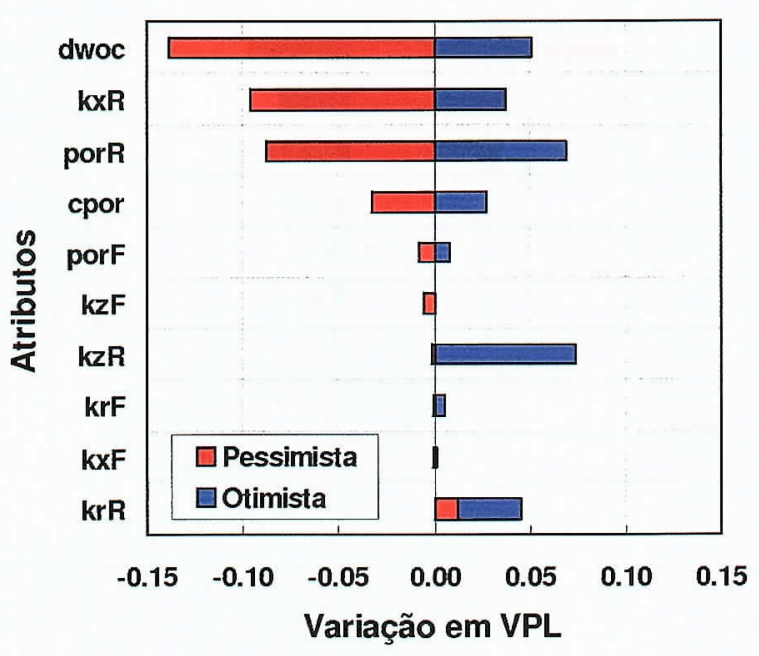

(a)

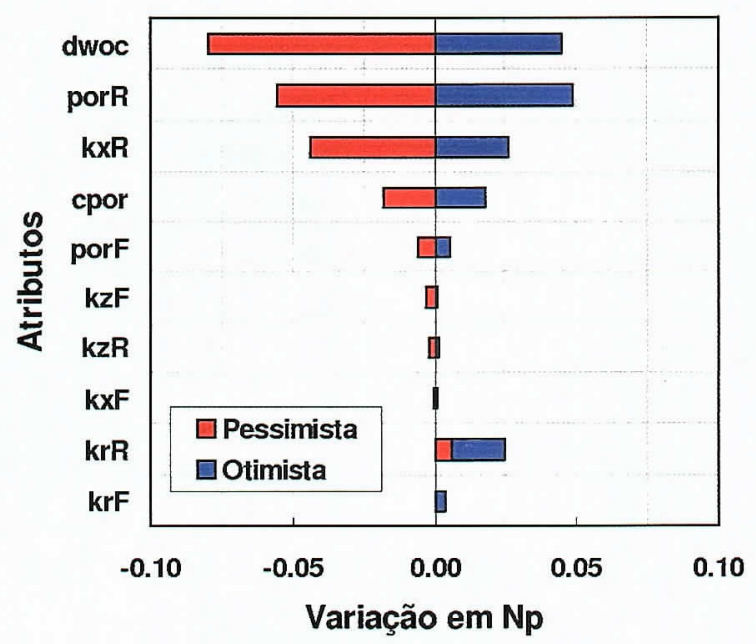

Figura 7 - Caso 2: Análise de sensibilidade para as funções-objetivo. (a) VPL e (b) Np.

do atributo cpor com seus três níveis de incerteza não resultou em alterações significativas na curva de risco do Caso 1, mas em contrapartida aumentou o esforço computacional e o tempo do processo, pois o número de modelos simulados cresceu exponencialmente com o aumento do número de atributos na árvore de derivação. Deste modo, no Caso 1, os atributos considerados como críticos foram $d w o c$ (contato óleo-água), $k x R$ (permeabilidade absoluta horizontal na fácies reservatório), por $R$ (porosidade na fácies reservatório) e $k r R$ (permeabilidade relativa água-óleo na fácies reservatório), todos com três níveis de incerteza.

Para o Caso 2, o efeito da adição gradativa de atributos nos valores dos percentis P10, P50 e P90 é apresentado na figura 10. Os atributos $d w o c, k x R$ e porR, com todos seus níveis de incerteza (otimista, provável e pessimista) foram adicionados à árvore de derivação, para o atributo $k z R$ apenas os níveis provável e otimista foram considerados, o que resultou num total de quatro atributos. O quinto e sexto atributos adicionados na árvore de derivação foram, respectivamente, $k r R$ (níveis provável e otimista) e cpor (três níveis de incerteza).

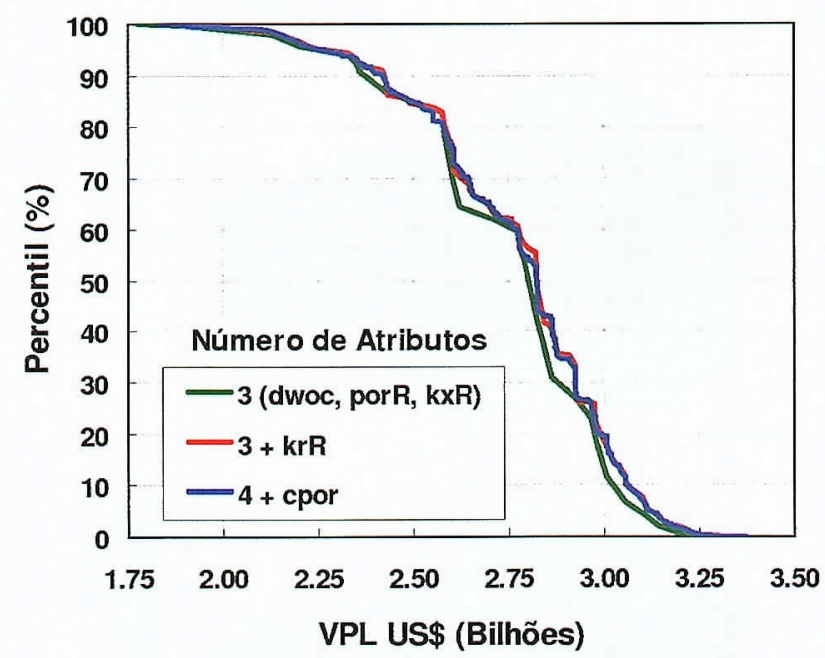

Figura 8-Caso 1: Curva de risco - adição gradativa de atributos incertos.

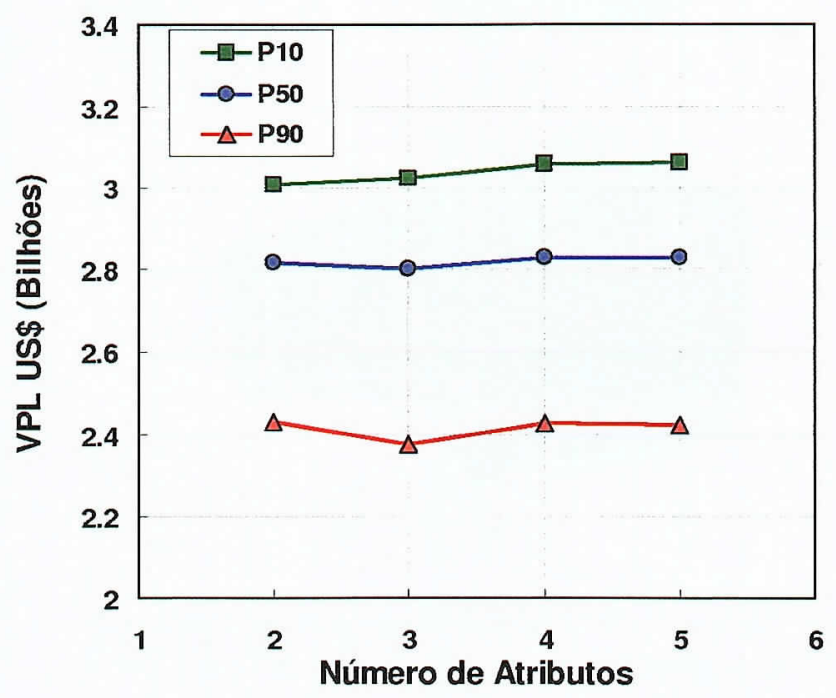

Figura 9-Caso 1: Efeito da adição gradativa de atributos nos valores dos percentis P10 (otimista), P50 (provável) e P90 (pessimista).

Observa-se que os valores do percentil P50 (provável) praticamente estabilizaram a partir da adição do quarto atributo. O mesmo não aconteceu para os percentis $\mathrm{P} 10$ (otimista) e P90 (pessimista). Tendo em mente o fato do crescimento exponencial do número de simulações com o aumento do número de atributos críticos, optouse em considerar para o Caso 2 cinco atributos críticos: $d w o c$ (contato óleo-água), $k x R$ (permeabilidade absoluta horizontal na fácies reservatório), por $R$ (porosidade na fácies reservatório), todos com três níveis de incerteza e $k z R$ (permeabilidade absoluta vertical na fácies reservatório) e $k r R$ (permeabilidade relativa água-óleo na fácies reservatório), ambos apenas com dois níveis de incerteza (provável e otimista).

Modelos Geológicos Representativos Uma vez determinados os atributos críticos e elaborada a curva de risco foram selecionados os modelos geológicos repre- 


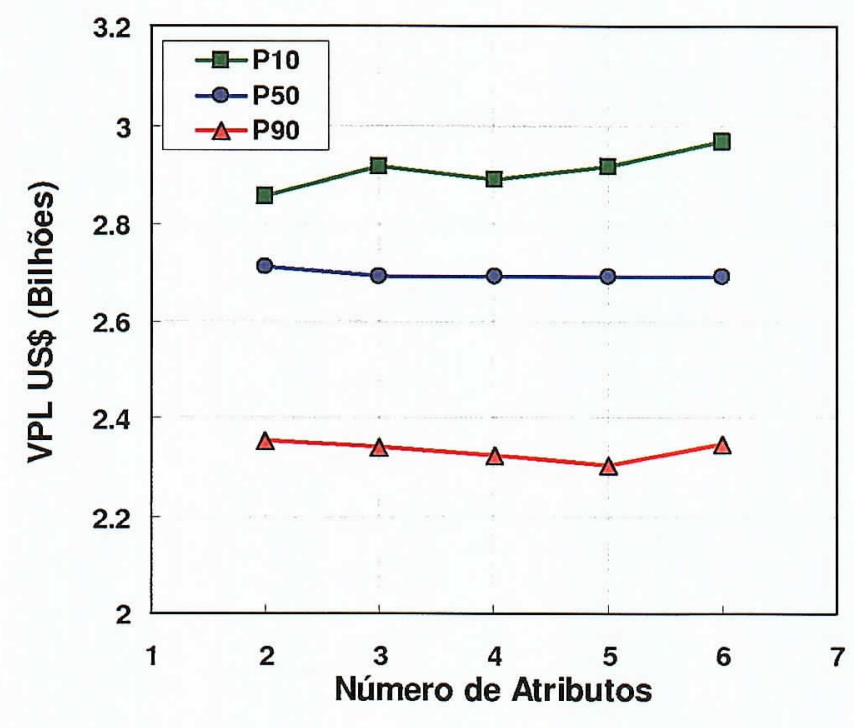

Figura 10 - Caso 2: Efeito da adição gradativa de atributos nos valores dos percentis: P10 (otimista), P50 (provável) e P90 (pessimista).

sentativos (MGR), os quais representam a variabilidade da estratégia de produção. A escolha dos MGR foi baseada em termos do indicador econômico VPL em função do fator de recuperação do óleo. Para ambos os Casos, um total de cinco modelos foi selecionado como MGR, sendo que o próprio modelo base constituiu um dos MGR, figura 11 e 12, respectivamente.

Árvores de Derivação e Decisão A transformação da árvore de derivação em árvore de decisão depende do número de atributos incertos que podem ter sua incerteza reduzida pela aquisição da informação. Nos casos em estudo a informação foi admitida como perfeita, ou seja, capaz de eliminar totalmente a incerteza associada ao atributo contato água-óleo. A elaboração da árvore de derivação deve seguir a Etapa 4 da metodologia, enquanto que sua transformação em árvore de decisão segue a Etapa 7.

O Caso 1 por conter um menor número de atributos críticos foi selecionado para ilustrar a árvore de derivação (Fig. 13), e sua transformação em árvore de decisão (Fig. 14).

Cálculo do Valor de Informação A otimização das estratégias de produção dos MGR é uma etapa muito importante para o cálculo do VDI, pois quanto melhor e mais eficiente a otimização de cada modelo, maior é a precisão e a confiabilidade do VDI calculado. A otimização de cada modelo é um processo dispendioso devido ao número de parâmetros que devem ser alterados e ao elevado número de simulações que devem ser realizadas a fim de maximizar uma ou mais funçõesobjetivo.

Os modelos geológicos representativos (MGR) dos Casos 1 e 2 foram submetidos à otimização de suas estratégias de produção. Embora possa haver flexibilidade na otimização de uma estratégia de produção,

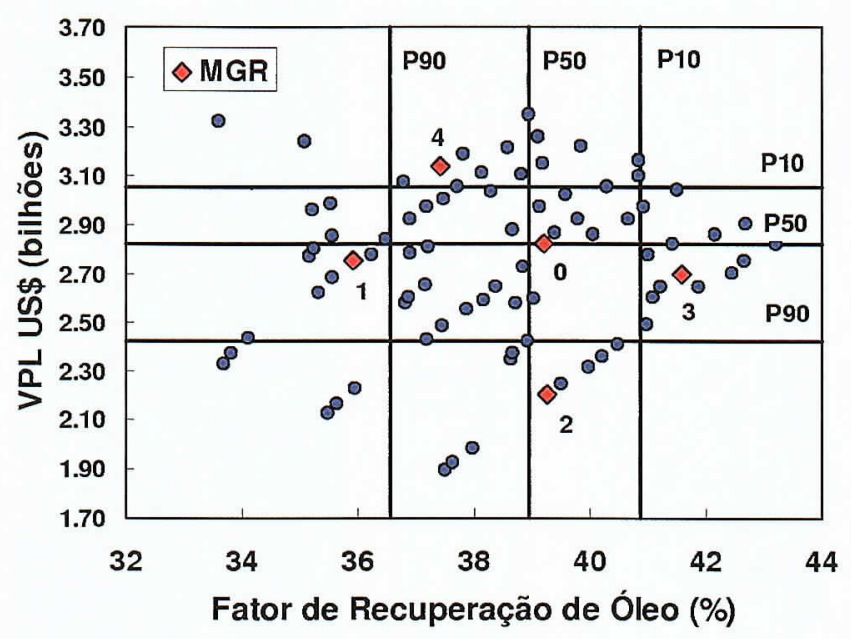

Figura 11 - Caso 1: Seleção dos Modelos Geológicos Representativos (MGR).

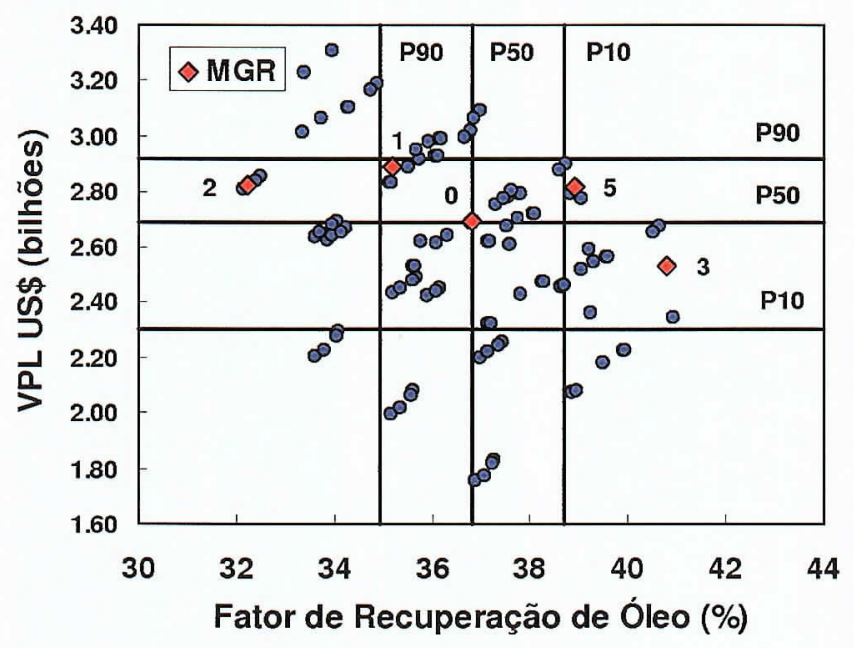

Figura 12 - Caso 2: Seleção dos Modelos Geológicos Representativos (MGR).

em ambos os casos estudados, neste trabalho, apenas o número e a posição dos poços foram alterados durante o processo de otimização dos MGR (a existência de maior flexibilidade de mudanças pode aumentar o VDI calculado). A tabela 2 mostra, para os Casos 1 e 2 , o número de poços produtores e injetores de cada MGR otimizado.

De posse dos MGR otimizados em termos da função-objetivo VPL, os modelos referentes às árvores de derivação dos Casos 1 e 2 foram simulados com cada uma das cinco estratégias de produção referentes aos seus respectivos MGR.

A estratégia de produção que resultou no maior VME para o Caso 1 e para o Caso 2 foi selecionada para representar o caso sem informação, ou seja, para determinar o $\mathrm{VME}_{\mathrm{SI}}$. É importante ressaltar que uma única estratégia de produção foi selecionada para determinar o $\mathrm{VME}_{\mathrm{SI}}$ de cada caso. Com o conhecimento da informação, para cada um dos três grupos da árvore de decisão dos Casos 1 e 2 foi determinada a estratégia 


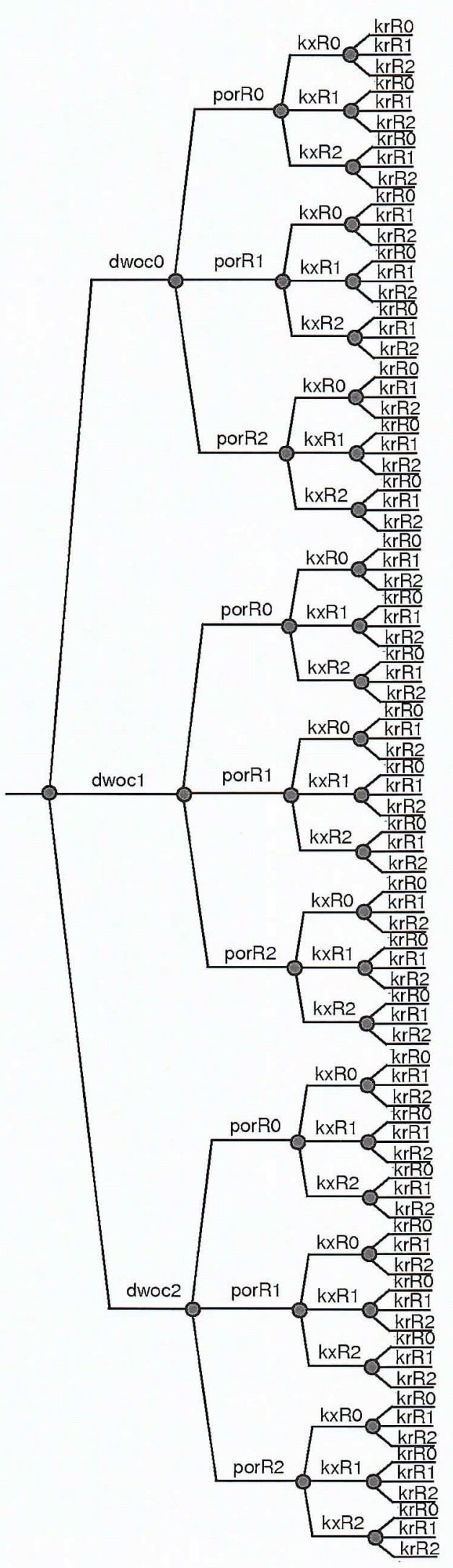

Figura 13 - Caso 1: Árvore de derivação com 4 atributos críticos.

de produção dentre as estratégias dos MGR capaz de maximizar o VME de cada grupo, cuja soma resultou no determinação do $\mathrm{VME}_{\mathrm{Cr}}$.

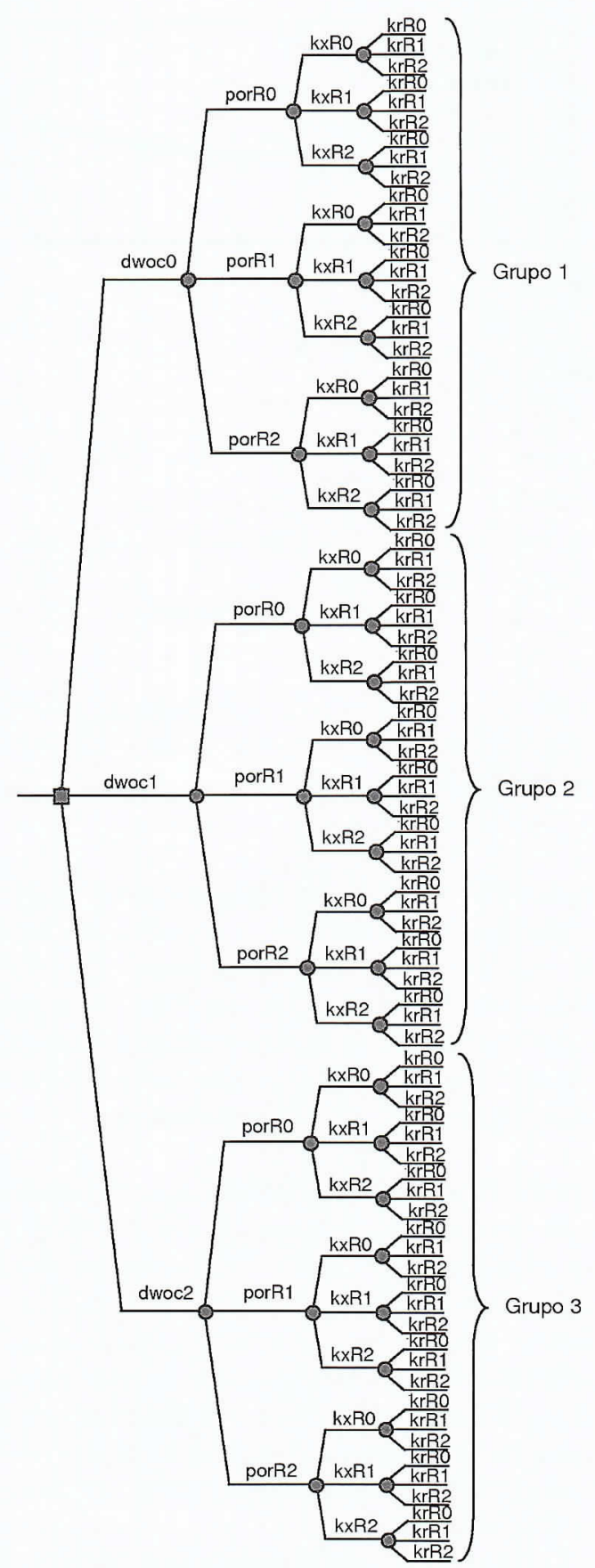

Figura 14 - Caso 1: Árvore de decisão com informação completa para o contato óleo-água (dwoc).

CASO 1 A tabela 3 mostra que a estratégia de produção do MGR 4 resultou no maior valor de VME para o caso sem informação $\left(\mathrm{VME}_{\mathrm{SI}}\right)$. Esta mesma estratégia, também, maximizou o VME de todos os três grupos da árvore de decisão. Conseqüentemente, o Valor da Informação obtido foi nulo. Contudo, isto não significa que a obtenção de uma informação adicional, como a perfuração de um poço adicional, possui custo nulo. Este resultado foi decorrente do fato de não se ter tirado o proveito adequado da informação adicional no procedimento de otimização dos MGR, ressaltando a 
Tabela 2 - Número de poços após a otimização dos $M G R$.

\begin{tabular}{c|c|c|c|c}
\hline & \multicolumn{2}{|c|}{ Caso 1 } & \multicolumn{2}{c}{ Caso 2 } \\
\hline \multirow{2}{*}{ Cenário } & $\begin{array}{c}\text { N. }{ }^{\circ} \text { de } \\
\text { Produtores }\end{array}$ & $\begin{array}{c}\mathrm{N} .{ }^{\circ} \mathrm{de} \\
\text { Injetores }\end{array}$ & $\begin{array}{c}\mathrm{N} .{ }^{\circ} \mathrm{de} \\
\text { Produtores }\end{array}$ & $\begin{array}{c}\mathrm{N} .{ }^{\circ} \mathrm{de} \\
\text { Injetores }\end{array}$ \\
\hline MGR 0 & 11 & 16 & 11 & 8 \\
\hline MGR 1 & 11 & 14 & 13 & 9 \\
\hline MGR 2 & 14 & 12 & 12 & 9 \\
\hline MGR 3 & 16 & 11 & 14 & 8 \\
\hline MGR 4 & 16 & 14 & 18 & 14 \\
\hline
\end{tabular}

um poço adicional para eliminar a incerteza do contato água-óleo foi de 17,68 milhões US\$. Portanto, neste caso, na otimização dos MGR tirou-se o proveito adequado da informação, de modo que o VDI apresentou valor maior que zero.

DISCUSSÃO Conforme mostrado por Xavier (2004) e Hayashi (2006), o VDI cresce com o aumento do número de MGR, com o aumento no número de simulações e com a sofisticação do processo de otimização, por isso, o VDI obtido é, em geral, subestimado. No Caso 1, o VDI zero não indica necessariamente que a informação não tem valor, mas que seu valor é baixo e

Tabela 3 - Caso 1: Determinação do $V M E_{S P} V M E_{C I}$ e VDI após otimização dos $M G R$.

\begin{tabular}{|c|c|c|c|c|c|c|}
\hline & & & US\$ & hões) & & \\
\hline Estratégia Otimizada para o Cenário & $\begin{array}{c}\text { VME } \\
\text { Grupo } 1 \\
\end{array}$ & $\begin{array}{c}\text { VME } \\
\text { Grupo } 2 \\
\end{array}$ & $\begin{array}{c}\text { VME } \\
\text { Grupo } 3 \\
\end{array}$ & $\mathrm{VME}_{\mathrm{SI}}$ & $\mathrm{VME}_{\mathrm{Cl}}$ & $\mathrm{VDI}^{(1)}$ \\
\hline MGR 0 & 1690.92 & 485.96 & 595.35 & \multirow{5}{*}{4213.69} & \multirow{5}{*}{4213.69} & \multirow{5}{*}{0} \\
\hline MGR 1 & 1726.60 & 509.00 & 603.46 & & & \\
\hline MGR 2 & 1685.53 & 491.06 & 600.57 & & & \\
\hline MGR 3 & 2318.22 & 675.28 & 838.53 & & & \\
\hline MGR 4 & $2544.02 *$ & $744.19 *$ & $925.48 *$ & & & \\
\hline
\end{tabular}

onde: * Valores de $\mathrm{VME}$ dos grupos usados para calcular $\mathrm{VME}_{\mathrm{CI}}$

(1) $\mathrm{VDI}=\mathrm{VME}_{\mathrm{CI}}-\mathrm{VME}_{\mathrm{Cl}}$

Tabela 4-Caso 2: Determinação do $V M E_{S P} V M E_{C I}$ e VDI após otimização dos $M G R$.

\begin{tabular}{|c|c|c|c|c|c|c|}
\hline \multirow[b]{2}{*}{ Estratégia Otimizada para o Cenário } & \multicolumn{6}{|c|}{ US\$ (Milhões) } \\
\hline & $\begin{array}{c}\text { VME } \\
\text { Grupo } 1\end{array}$ & $\begin{array}{c}\text { VME } \\
\text { Grupo } 2\end{array}$ & $\begin{array}{c}\text { VME } \\
\text { Grupo } 3\end{array}$ & $\mathrm{VME}_{\mathrm{SI}}$ & $\mathrm{VME}_{\mathrm{CI}}$ & $\mathrm{VDI}^{(1)}$ \\
\hline MGR 0 & 1614.45 & 460.46 & 570.43 & \multirow{5}{*}{3226.47} & \multirow{5}{*}{3244.10} & \multirow{5}{*}{17.63} \\
\hline MGR 1 & 1887.63 & 552.53 & 666.14 & & & \\
\hline MGR 2 & 1870.12 & 505.47 & 685.08 & & & \\
\hline MGR 3 & 1880.38 & $557.65^{*}$ & 649.17 & & & \\
\hline MGR 4 & $1960.08^{*}$ & 540.02 & $726.37 *$ & & & \\
\hline
\end{tabular}

importância e necessidade de utilizar adequadamente as informações adicionais na otimização das estratégias de produção dos MGR.

CASO 2 A tabela 4, referente ao Caso 2, mostra que a estratégia de produção do MGR 4 resultou no maior valor de VME para o caso sem informação $\left(\mathrm{VME}_{\mathrm{SI}}\right)$. No cálculo do VME com informação $\left(\mathrm{VME}_{\mathrm{CI}}\right)$, os maiores valores de VME para os Grupos 1, 2 e 3 foram, respectivamente, obtidos pelas estratégias de produção dos MGR 4, MGR 3 e MGR 4. O custo da perfuração de próximo de zero; isso significa, para o Caso 1 estudado, que a informação não traz grandes benefícios para a decisão de número e posição dos poços. No Caso 2 , como o VDI é subestimado, pode-se afirmar que se o custo da informação for inferior a 17,63 milhões US\$, devese obter a informação adicional. Contudo, se o custo da informação for um pouco superior ao VDI obtido, faz-se necessário sofisticar o processo, melhorando a otimização das estratégias de produção dos MGR ou aumentando o número de MGR selecionados. Se esse custo for muito superior, pode-se descartar essa possi- 
bilidade:

CONCLUSÕES Os dois casos estudados mostraram ser bons exemplos para ilustrar a metodologia de cálculo do Valor Monetário sem Informação $\left(\mathrm{VME}_{\mathrm{SI}}\right)$ e com Informação $\left(\mathrm{VME}_{\mathrm{Cl}}\right)$ os quais possibilitam avaliar a viabilidade econômica da obtenção de uma ou mais informações adicionais a fim de eliminar ou reduzir incertezas associadas a reservatórios de petróleo na fase de desenvolvimento. Um dos casos mostrou que é possível obter um VDI nulo e como qualquer informação adicional está associada a um custo, deve-se ter cautela na aquisição da informação, pois ela pode não trazer benefícios para o projeto. Um VDI nulo também é um indicador de que os benefícios referentes à informação adicional não foram devidamente explorados no processo de otimização dos Modelos Geológicos Representativos (MGR). Pode-se mudar este valor através de uma maior flexibilidade na estratégia de produção para que se possa tirar proveito da informação. O segundo caso estudado, no qual tirou-se o proveito adequado da informação adicional na otimização dos MGR, resultou num VDI próximo do custo da perfuração de um poço adicional. Como o VDI é em geral subestimado, podese afirmar que é interessante adquirir a informação. A precisão e confiabilidade do VDI resultam da otimização de estratégias de produção dos MGR. Quanto maior o número de MGR e mais eficiente for a otimização da estratégia de cada MGR, maior é precisão e maior a confiabilidade no valor de VDI. De posse de um valor confiável de VDI, o executor do projeto optará ou não pela aquisição de uma nova informação.

Agradecimentos Os autores agradecem a Petrobras (Rede SIGER) e ao grupo de pesquisas UNISIM (CEPETRO/UNICAMP).

\section{Referências}

Begg S., Bratvold R, Campbell J. 2002. The Value of Flexibility in Managing Uncertainty in Oil and Gas Investments. In: SPE, Annual Technical Conference and Exhibition (ATCE), SPE 77586, USA, 1 CD-Rom.

Clemen R.T., 1995. Making Hard Decisions - An Introduction to Decision Analysis. Belmont, 664p.

Costa A.P.A \& Schiozer D.J. 2003. Treatment of Geological Attributes in Risk Analysis Applied to the Appraisal Phase of Petroleum Fields. In: Canadian International Petroleum Conference, Calgary, Alberta, Canada, 1 CDRom.

Coopersmith E.M. \& Cunningham M.P.C., 2002. A Practical Approach to Evaluating the Value of Information and Real Option Decisions in the Upstream Petroleum Industry. In: SPE, Annual Technical Conference and Exhibition (ATCE), SPE 77582, EUA, 1 CD-Rom.

Demirmen F. 2001. Subsurface Appraisal: The Road from Reservoir Uncertainty to Better Economics. In: SPE, Hydrocarbon Economics and Evaluation Symposium, Dallas, U.S.A, SPE 68603, 1 CD-Rom.

Demirmen F., 1996. Use of value of Information Concept in Justification and Ranking of Subsurface Appraisal. In: SPE, Annual Technical Conference and Exhibition (ATCE), Denver, USA, SPE 36631, 1 CD-Rom.

Floris F.J.T. \& Peersmann M.R.H.E. 2000. E\&P Decision System for Asset Management - A case study. SPE European Petroleum Conference, SPE 65146, Paris, França, 1 CD-Rom.

Hayashi S.H.D. 2006. Valor da Flexibilização e Informação em Desenvolvimento de Campo por Módulos. Dissertação de Mestrado em Ciências e Engenharia de Petróleo, FEM e IG, UNICAMP, Campinas, 118 p.

Hayashi S.H.D., Ligero E.L., Schiozer D.J. 2007. DecisionMaking Process in Development of Offshore Petroleum Fields, SPE 107737. In: SPE Latin American and Caribbean Petroleum Engineering Conference, Buenos Aires, Argentina, 1 CD-Rom.

Koninx J.P.M., 2000. Value of Information: From Cost Cut- ting to Value Creation. In: SPE, Asia Pacific Oil and Gas Conference and Exhibition, SPE 64390, Australia, 1 CDRom.

Maschio C., Vidal, A.C., Schiozer, D.J. (submetido). A Framework to Integrate History Matching and Geostatistical Modeling using Genetic Algorithm and Direct Search Methods Journal of Petroleum Science and Engineering.

Mian M.A. 2002. Project economics and decision analysis. Tulsa, Okla., Penn well, 411 p.

Santos J.A.M. \& Schiozer D.J. 2003. Quantifying Production Strategy Impact in Risk Analysis of an E\&P Project Using Reservoir Simulation. In: Reservoir Simulation Symposium, $17^{\text {th }}$, Houston, EUA, SPE 79679, 1 CD-Rom.

Schiozer D.J., Ligero E.L., Suslick S.B., Costa A.P.A., Santos J.A.M. 2004. Use of Representative Models in the Integration of Risk Analysis and Production Strategy Definition. Journal of Petroleum Science and Engineering, 44(1/2):131-141.

Steagall D.E. \& Schiozer D.J. 2001. Uncertainty Analysis in Reservoir Production Forecast during the Appraisal and Pilot Production Phases. In: SPE Reservoir Simulation Symposium, SPE 66399, EUA, 1 CD-Rom.

Stermole F.J. \& Stermole J.M. 1974. Economic Evaluations and Investment Decision Methods. Colorado, Golden: Investment Evaluation Corporation, 353p.

Xavier A.M. 2004. Análise do Valor da Informação na Avaliação e Desenvolvimento de Campos de Petróleo. Dissertação de Mestrado em Ciências e Engenharia de Petróleo, FEM e IG, UNICAMP, Campinas, 160p.

Xavier A.M., Ligero E.L., Schiozer D.J., 2006. Metodologia para Cálculo do Valor de Informação no Desenvolvimento de Campos de Petróleo. In: Rio Oil and Gas Expo and Conference, Rio de Janeiro, 1 CD-Rom.

Manuscrito BR 07 Submetido em 21 de dezembro de 2007 Aceito em 10 de março de 2008 